\title{
Hybrid GaSb/Si swept-wavelength laser sensor technology for next generation wearable healthcare device platform
}

Augustinas Vizbaras, leva Šimonytè, Arūnas Miasojedovas, Tadas Bučiūnas, Greta Naujokaitè, et al.

Augustinas Vizbaras, leva Šimonytè, Arūnas Miasojedovas, Tadas Bučiūnas, Greta Naujokaitè, Mindaugas Greibus, Augustinas Trinkūnas, Andreas De Groote, Daan Martens, Žilvinas Dambrauskas, Antanas Gulbinas, Gunther Roelkens, Kristijonas Vizbaras, "Hybrid GaSb/Si swept-wavelength laser sensor technology for next generation wearable healthcare device platform," Proc. SPIE 10885, Optical Diagnostics and Sensing XIX: Toward Point-ofCare Diagnostics, 108850T (20 February 2019); doi: 10.1117/12.2508471 


\title{
Hybrid GaSb/Si swept-wavelength laser sensor technology for next generation wearable healthcare device platform
}

\author{
Augustinas Vizbaras ${ }^{\mathrm{a}^{*}}$, Ieva Šimonytè ${ }^{\mathrm{a}}$, Arūnas Miasojedovas ${ }^{\mathrm{a}}$, Tadas Bučiūnas ${ }^{\mathrm{a}}$, Greta Naujokaitė ${ }^{\mathrm{a}}$ \\ Mindaugas Greibus $^{\mathrm{a}}$, Augustinas Trinkūnas ${ }^{\mathrm{a}}$, Andreas De Groote ${ }^{\mathrm{b}}$, Daan Martens ${ }^{\mathrm{b}}$, Žilvinas \\ Dambrauskas $^{c}$, Antanas Gulbinas ${ }^{c}$, Gunther Roelkens ${ }^{\mathrm{d}}$, and Kristijonas Vizbaras ${ }^{\mathrm{a}}$ \\ ${ }^{a}$ Brolis Semiconductors UAB, Moletu pl. 73, LT-14259 Vilnius, Lithuania \\ ${ }^{\mathrm{b}}$ Brolis Semiconductors BVBA, Bollebergen 2B Box 11, BE-9052 Ghent, Belgium \\ ${ }^{\mathrm{c}}$ Institute for Digestive Research, Medical Academy, Lithuanian University of Health Sciences, \\ Eiveniu g. 4, LT-50161 Kaunas, Lithuania \\ ${ }^{\mathrm{d}}$ Ghent University-Imec, Photonics Research Group (INTEC), Technologiepark-Zwijnaarde 126, \\ BE-9052 Ghent, Belgium \\ *corresponding author: augustinas.vizbaras@brolis-semicon.com
}

\begin{abstract}
Spectral region beyond $1.7 \mu \mathrm{m}$ is particularly interesting for biomedical spectroscopic sensing applications due to the presence of strong and molecule-specific ro-vibrational overtone and combination absorption bands for a number of important analytes such as glucose, lactate, urea, human serum albumin among others. However, this spectral region has been largely unexplored for applications targeting wearable device technology due to the absence of commercially available semiconductor light source technology. In this work we report on recent progress in developing beyond-stateof-the-art GaSb-based swept-wavelength laser technology as a key building-block of the proposed spectroscopic sensor concept. To demonstrate the capability of the technology, we provide experimental data of in vitro sensing concentrations down to the normal physiological range and beyond for glucose, lactates, urea and bovine serum albumin. Furthermore, we provide initial experimental evidence of non-invasive in vivo sensing experiment with extracted absorbance signature of human serum albumin collected from the wrist and demonstrate a clear path towards sensing other analytes. Finally, to demonstrate the full potential of the spectroscopic sensor technology for the wearable device market, we present results of our initial effort to realize a complete spectroscopic sensor system-on-a-chip based on hybrid $\mathrm{GaSb} / \mathrm{Si}$ material platform and manufactured using conventional $200 \mathrm{~mm}$ silicon-on-insulator CMOS technology process in a commercial high-volume foundry.
\end{abstract}

Keywords: silicon photonics, gallium antimonide, glucose sensing, wearables, swept-wavelength laser, molecular beam epitaxy

\section{INTRODUCTION}

Wearable technologies have been attracting significant attention in the recent years fueled by the increasing developments in the technology miniaturization, progress in sensor technology and general awareness about the potential benefits to the consumer [1]. A particularly attractive route to market is to utilize the existing technology platforms, already adopted by millions of users across the world such as smartphones and smart watches [2] as all the other wearable technology interfaces such as smart-textiles, smart paper etc. will still need to overcome the barrier of user adoption which in consumer electronics is difficult to estimate. We argue that a significant market entry or adoption catalyzers would the usefulness of the additional feature in the existing product- i.e. the added value to the of the additional information provided by the sensor within the wearable. While current commercial devices provide some

Optical Diagnostics and Sensing XIX: Toward Point-of-Care Diagnostics, edited by Gerard L. Coté, Proc. of SPIE Vol. 10885, 108850T · C 2019 SPIE · CCC code: 1605-7422/19/\$18 · doi: 10.1117/12.2508471 
basic functionality such as pace calculation via integrated accelerometers, or, recently introduced, wrist-based heart rate monitoring offered by leading commercial vendors [3], the currently available commercial technology is still unable to provide adequate insight into the physiological condition of the person in order to drive the so-called personalized healthcare revolution [4]. The latter being a result of many reasons rather than one, from inadequate sensor performance when it comes to the user to the stringent manufacturability and cost per part requirements when it comes to consumer market and the product volume therein among others.

In this work, we provide our recent results in developing an integrated micro blood analyzer system-on-a-chip using hybrid $\mathrm{GaSb} / \mathrm{Si}$ material platform. GaSb-based gain-chip was chosen as the light source technology of choice as it can efficiently cover the 1.7-2.5 micron wavelength region. In addition, efficient room-temperature photodiodes are also available with good sensitivity up to about 2.6 micron, which is important for the system design. From the application point of view, this spectral region is particularly interesting for molecular sensing due to the presence of strong and molecule-specific ro-vibrational absorption bands. These absorption bands correspond to the first overtone of the $\mathrm{C}-\mathrm{H}$ stretching vibrations and the combination of stretching and bending vibrations of $\mathrm{C}-\mathrm{H}, \mathrm{N}-\mathrm{H}$ and $\mathrm{O}-\mathrm{H}$ bonds, acting as a unique molecular fingerprint for sensing applications and can be sensed by means of direct photon-phonon (target molecule vibration) interaction $[5,6]$ In essence, this means measuring direct response without any lag, drift or saturation as compared to existing electrochemical sensors such as point-of-care glucometers or electrochemical continuous glucose monitoring devices (CGM), where the glucose signal is monitored as a product of one or more enzymatic reactions [7]. In liquid phase such as whole blood or serum, these absorption bands are broadened due to molecular collisions with characteristic spectral shape spanning over $100 \mathrm{~nm}$ of bandwidth [5, 6], therefore optical sensing requires a light source with a spectral bandwidth of at least the same width. This can be achieved by using a broadband source such as a blackbody emitter or a broadband LED, which however suffers from poor performance and low efficiency in the spectral region beyond 1.7 micron and provides low spectral power density, which limits the overall sensor sensitivity. An alternative approach is to use a broadly tunable laser source, which could sweep across a bandwidth of $100 \mathrm{~nm}$ or more and cover the complete absorption spectrum of the molecule of interest. In practical sensing scenario, typical concentrations of relevant biomolecules such as glucose, lactate, urea in physiologically relevant substance such as blood, serum or interstitial fluid are in fairly low and correspond to several $\mathrm{mmol} / \mathrm{l}$, which in turns lead to a sensing signal change of the order of $0.01 \%$ and thus requires as high signal to noise ratio as possible [5]. Due to the narrow linewidth and the ability perform the wavelength sweep at high speed, swept-wavelength laser (SWL) approach provides the best possible spectral power density and thus highest signal to noise ratio.

In this paper we address the use of SWL technology for biomedical sensing applications and present results with several relevant biomolecules as well as describe the path toward miniaturization, manufacturability of the technology

The paper is divided into separate sections, first describing the key enabling hardware technology, spectroscopic sensing results and finalizing with an outlook towards the next generation sensor technology for wearables.

\section{GaSb SWEPT-WAVELENGTH LASER TECHNOLOGY}

\subsection{Key component: GaSb type-I gain-chip}

In this work, the described swept-wavelength laser (SWL) concept is based on a GaSb based type-I quantum well (QW) gain-chip embedded in external cavity laser configuration. Laser wavelength tuning is achieved either by changing the deflection angle of the MEMS mirror, when the sensor is realized as a table top replica for application validation [5] or by electrothermal tuning of Vernier-filter when the external cavity is realized within a photonic integrated circuit (PIC) $[9,10]$. Gain-chip is a key element as it provides optical gain for the laser and defines the tuning bandwidth.

The device wafers were epitaxially grown on 3-inch Te doped $n+$ GaSb substrates using an industrial solid-source multiwafer molecular beam epitaxy machine Veeco Gen200Edge, equipped with Al, Ga, In, As, Sb as elemental source materials and $\mathrm{Si}, \mathrm{GaTe}$ and $\mathrm{Be}$ as dopant sources. Quantum well composition and thickness were changed to tune the emission wavelength. For $1.7 \mu \mathrm{m}$ wavelength, AlGaInSb QWs were used in the active region. For 1.9 and $2.1 \mu \mathrm{m}$ emission wavelengths, GaInSb QW material was used. For longer wavelengths, quaternary GaInAsSb QWs were used. 
QW and waveguide thicknesses were adjusted for a $1 \%$ transverse vertical optical confinement factor per QW. Detailed device epitaxial structure can be found in [5]. The wafers were then processed as quasi-index guided single-angled facet (SAF) gain-chip devices. For a single spatial mode output, the $4 \mu \mathrm{m}$-wide and $\sim 1.5 \mu \mathrm{m}$-deep ridge waveguides were defined by UV photolithography and Cl-based ICP RIE etching, followed by sputtering of a $~ 330 \mathrm{~nm}$ thick $\mathrm{SiO}_{2}$ insulation. A contact window on the top of the ridge was defined by lithography and opened by means of $\mathrm{CF}_{4} / \mathrm{O}_{2}$ based ICP-RIE dry etching. Ti/Pt/Au was used as a top ohmic contact followed by a $\sim 2 \mu \mathrm{m}$ thick Au plated heatsink. The wafers were thinned down to $\sim 120 \mu \mathrm{m}$ to facilitate cleaving. As the last step, the back side $\mathrm{Ti} / \mathrm{Pt} / \mathrm{Au}$ contact was evaporated. The wafers were cleaved into bars of $1 \mathrm{~mm}$ and $0.7 \mathrm{~mm}$ cavity length and the bar facets were coated. The output facet was anti-reflection coated to have the reflectivity $<0.1 \%$ and the back facet got a high reflectivity coating with the reflectivity $>95 \%$. Figure 1 illustrates microscope image of the $\mathrm{GaSb}$ gain-chip.

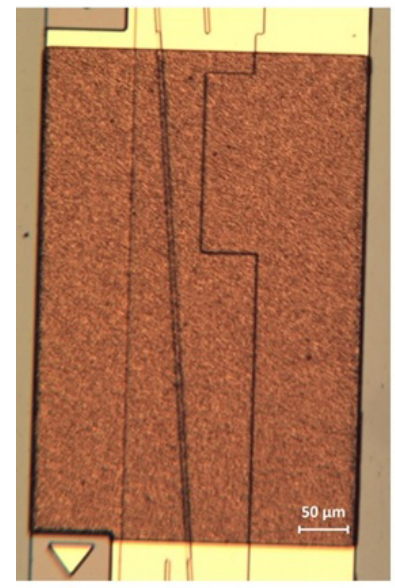

(a)

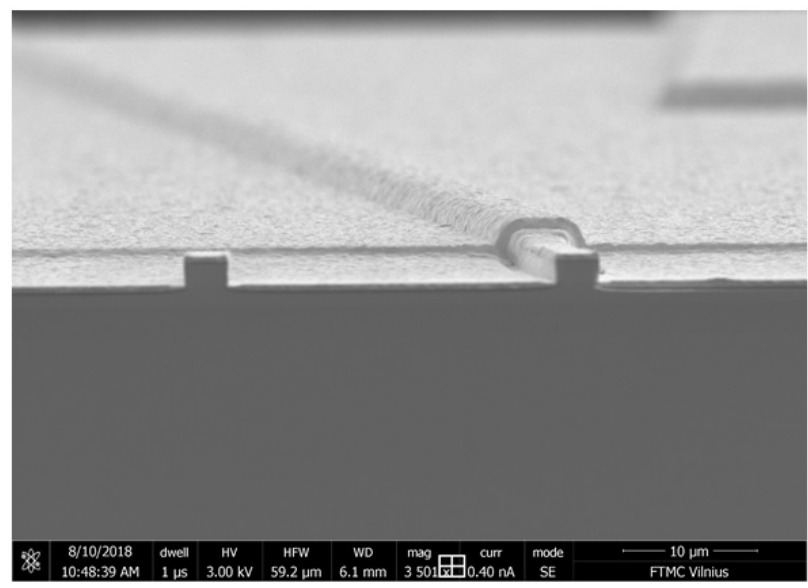

(b)

Fig.1 (a) Optica microscope image of a typical GaSb type-I gain-chip; (b) SEM image of the output facet of the processed chip.

\subsection{Swept-wavelength laser concept}

Swept-wavelength laser described in this work addresses a laser that is able to sweep the wavelength across the gainbandwidth rapidly. For the application validation, we used the gain-chip embedded in a miniature MEMS-driven Metcalf-Littman configuration (Fig 2a). Here, a standard off-the-shelf holographic grating with 600 grooves $/ \mathrm{mm}$ and $1200 \mathrm{~nm}$ blaze angle was used in combination with a commercial short-focal length and high NA aspheric lens for the beam collimation. The electrically driven MEMS mirror with a mechanical resonant frequency of around $200 \mathrm{MHz}$ was used enabling useful operation up to $150 \mathrm{~Hz}$. For continuous operation the MEMS mirror was driven by a sine function, effectively covering 300 sweeps/s for backward and forward MEMS mirror motion. For the fully integrated device, the swept-wavelength laser is also based on external cavity configuration, however this time the external cavity is define in the silicon chip. Wavelength filtering is performed by means of Vernier-filter and a reflector (Fig. 2b). As can be seen from Fig. 2- silicon photonics offers extreme size scaling. Even though our developed MEMS based SWL is compact compared to existing table top external cavity lasers, the hybrid $\mathrm{GaSb} / \mathrm{Si} \mathrm{SWL}$ offers a footprint 14000 smaller and can be tuned without moving parts. Moreover, the integrated laser is compatible with the technology of scale as both $\mathrm{GaSb}$ and Si parts can be easily scaled due to the nature of semiconductor technology. This is critical in the application theaters such as wearable consumer devices. Not only has the sensor be small or low-power consumption but most importantly it has to be very low-cost. 


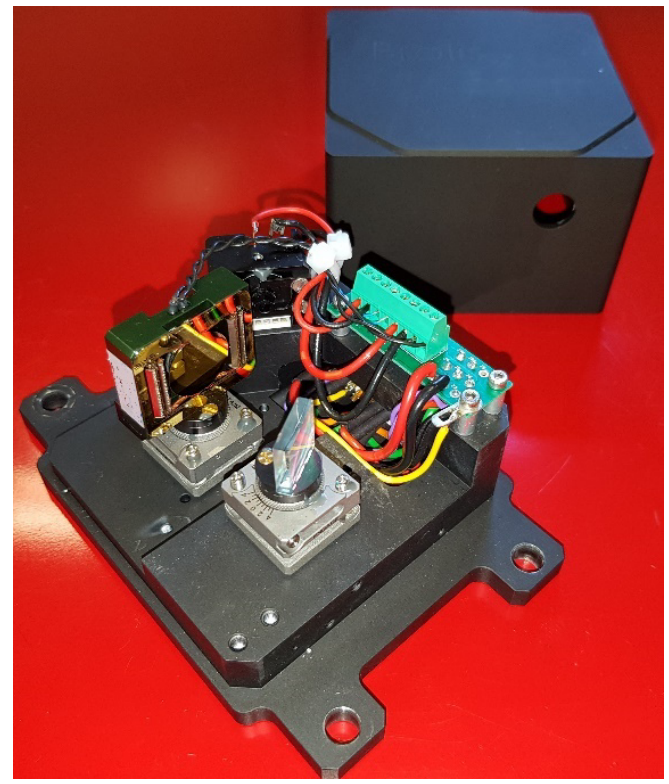

(a)

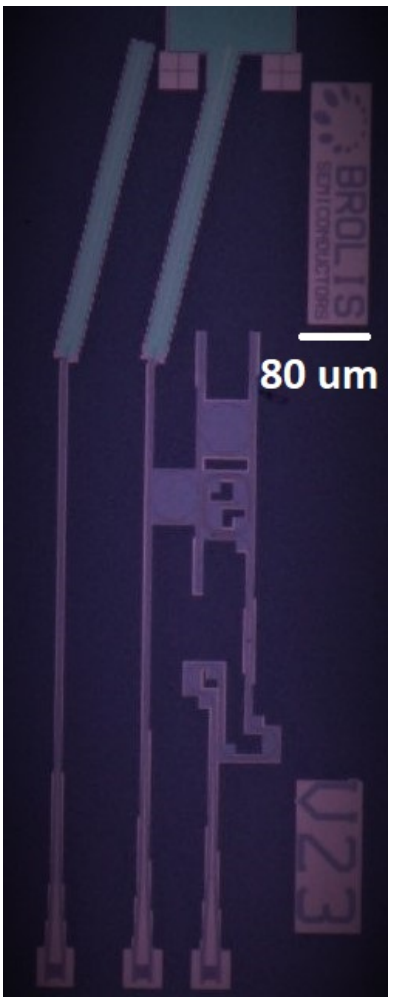

(b)

Fig.2. (a) A miniature table-top MEMS-driven Littmann-Metcalf external cavity diode swept-wavelength laser used for gain-chip technology optimization as well as application validation experiments. The dimensions $(\mathrm{WxLxH})$ are 75 mm x $110 \mathrm{~mm}$ x $54 \mathrm{~mm}$. (b) A SWL laser realized with silicon photonics technology. Overall footprint $\sim 1 \mathrm{~mm} \times 0.3$ $\mathrm{mm}$.

When constructing a spectroscopic sensor in the 1.7-2.5 micron band, the spectral width of the target molecule needs to be known in order to choose the appropriate number of gain-chips and thus SWLs within the sensor. Our GaSb type-I gain-chips provide extremely wide optical gain while still maintaining high output power. Figure 3 illustrates two gainchips of different design embedded in MEMS based SWL. An extremely wide laser sweep span can be seen in excess of $300 \mathrm{~nm}$, which correspond to almost $600 \mathrm{~cm}^{-1}$ from a single gain-chip. In addition, the output power is maintained equal to or higher than $20 \mathrm{~mW}$ in $\mathrm{CW}$ mode. To the best of our knowledge this is the broadest tuning bandwidth reported from a single composition not-cascaded or chirped type-I quantum well based gain-chip. The availability of such gain-chip performance allows considering a spectroscopic sensor that could target multiple molecules using a single gain-chip. In addition, MEMS mirror allows rapid wavelength sweep - i.e. up to $150 \mathrm{~Hz}$ in our case, which allow a wavelength sweep rate of $180000 \mathrm{~cm}^{-1} / \mathrm{s}$. This is particularly beneficial in case of measuring diffuse and turbulent substances such as whole blood or performing in-vivo measurements through skin. Rapid sampling allows minimizing the effect of the sample degradation a nd change with time (for instance blood clotting) or physiological undesired effects such as trembling, moving, shaking etc. With the described sweep rate, real time monitoring can be performed.

For the integrated sensor architecture, wavelength sweeping is performed by electro-thermally tuning the Vernier-filter. This has an advantage of being monolithic without any moving parts. Furthermore, the tuning rate can go in excess of $100 \mathrm{kHz}$ depending on the actual design and the thermal properties of the chip and the heater technology. 


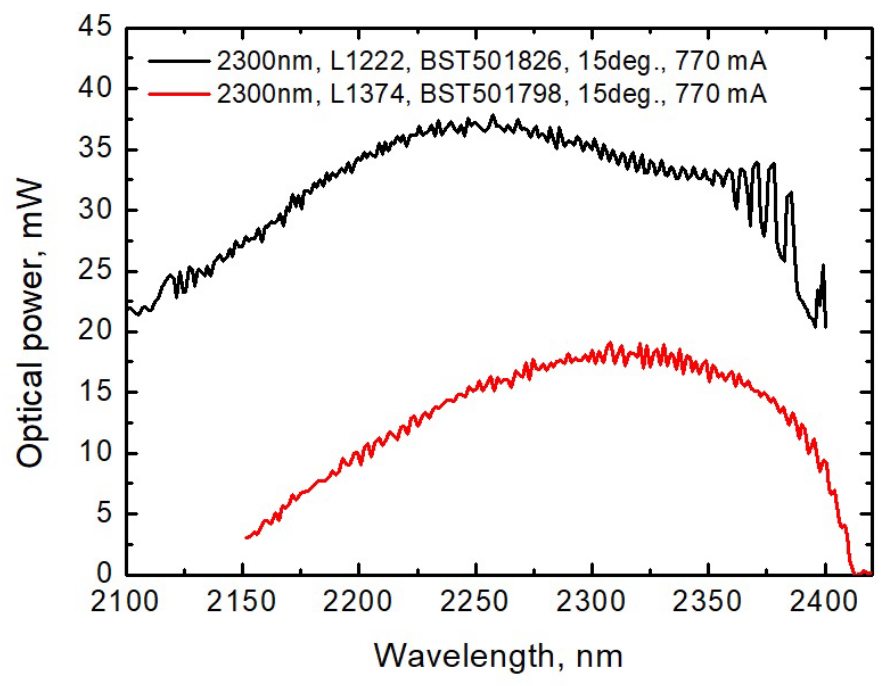

Fig. 3. Tuning curves of two swept-wavelength laser based on two different design gain-chips ( L1222 and L1274). Experimental conditions were $15 \mathrm{deg} \mathrm{C}$ and the drive current was $770 \mathrm{~mA}$. MEMS mirror drive frequency was 150 $\mathrm{Hz}$.

\section{SPECTROSCOPIC SENSOR}

\subsection{Concept and preliminary results}

Widely swept-wavelength laser is a key building block for a spectroscopic sensor. Such a sensor is particularly attractive for sensing critical biomarkers such as glucose, urea, lactate, etc. Among those, glucose is the most important molecule as it is relevant for many millions of people suffering from chronic diseases such as type-I and type-II diabetes. Typical absorption spectra of these molecules is shown in Fig 4.
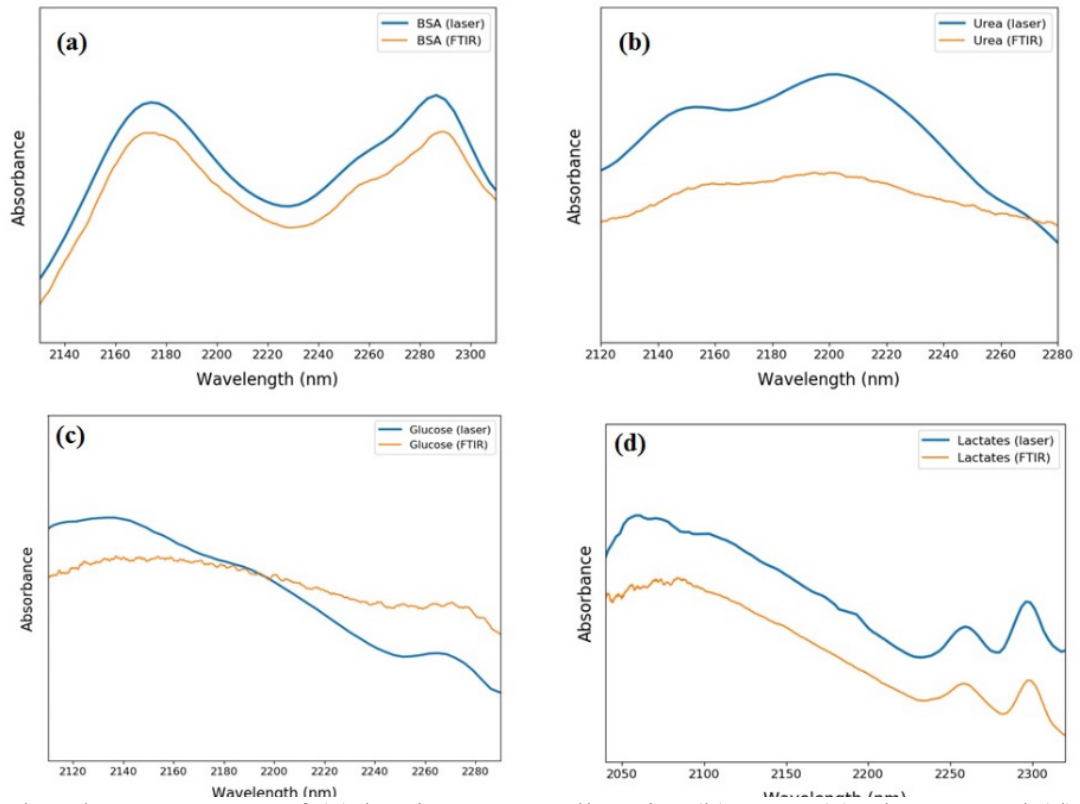

Fig. 4. Measured absorbance spectra of (a) bovine serum albumin, (b) urea, (c) glucose and (d) lactate 
It can be seen that a wavelength tuning bandwidth in excess of $150 \mathrm{~nm}$ is needed in order to fully resolve the spectral shape of the molecule-specific absorption spectrum. In practical sensing scenario such as sensing a physiological substance such as whole blood or blood serum, the molecule specific absorbance is very small compared to dominant background coming manly from water. In comparison, human serum albumin - the most dominant protein in human blood typically has absorbance change below $1 \%$ change compared to the baseline signal, whereas small molecules such as urea, lactate and glucose have their typical absorbance changes $<0.1 \%$. Therefore, wavelength stability needs to be accurately monitored as even small nonlinearities in the system can cover the "useful" signal from the molecule. Fig. 5 shows a schematic diagram of a SWL based spectroscopic sensor configured to perform in-vitro transmission measurements. In our work we combined the outputs of three SWLs based on three different design gain-chips to provide sufficient spectral overlap among the lasers as well as to enable sensing multiple molecules of interest as well as take into account water displacement due to temperature effects, allowing better control of the baseline signal. As per Fig 5 , the sensor would first provide a tunable wavelength light signal that would be transmitted through a sample with a cuvette. The wavelength and intensity of the SWL would be monitored by the wavelength-control block. The swept wavelength light would interact with the molecules within the cuvette and the modified spectrum would be collected with a single photodiode.

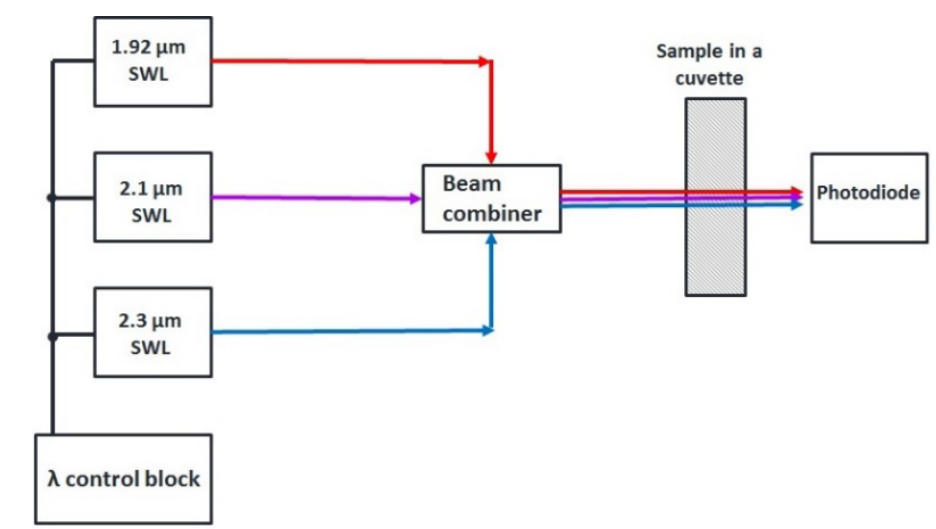

Fig. 5. A schematic diagram of the SWL-based spectroscopic sensor.

Obtained spectroscopic data would then be analyzed using statistical regression method based on multivariate partial least square algorithm constructed using known target molecule absorbance and corresponding concentration data to obtain a quantitative measurement. A particular advantage of using widely swept-wavelength lasers is the ability gather a large number of molecule specific wavelengths within one sweep, which is extremely important for the signal processing algorithms such as statistical regression based on multivariate partial least square algorithm. For example, in our case we use 150-200 specific wavelengths/molecule, allowing to decompose complex molecular substances. For example, in Fig.6 we perform sensing in a three-molecule based solution of tris-buffer saline (TBS), bovine serum albumin (BSA) and glucose. TBS and BSA represent strongest baseline components in blood - water and human serum albumin. Experimental sensor validation curve is shown in Fig. 6 collected from more than 100 data sets. 88 datasets were used to construct the algorithm and 33 randomly selected datasets were used as validation. It can be seen that excellent determination coefficient R2 value of $99 \%$ is obtained with a Root-Mean-Square-Error-of-Prediction of $0.324 \mathrm{mmol} / \mathrm{l}$ demonstrating the potential of the GaSb-based SWL spectroscopic sensing technology. 


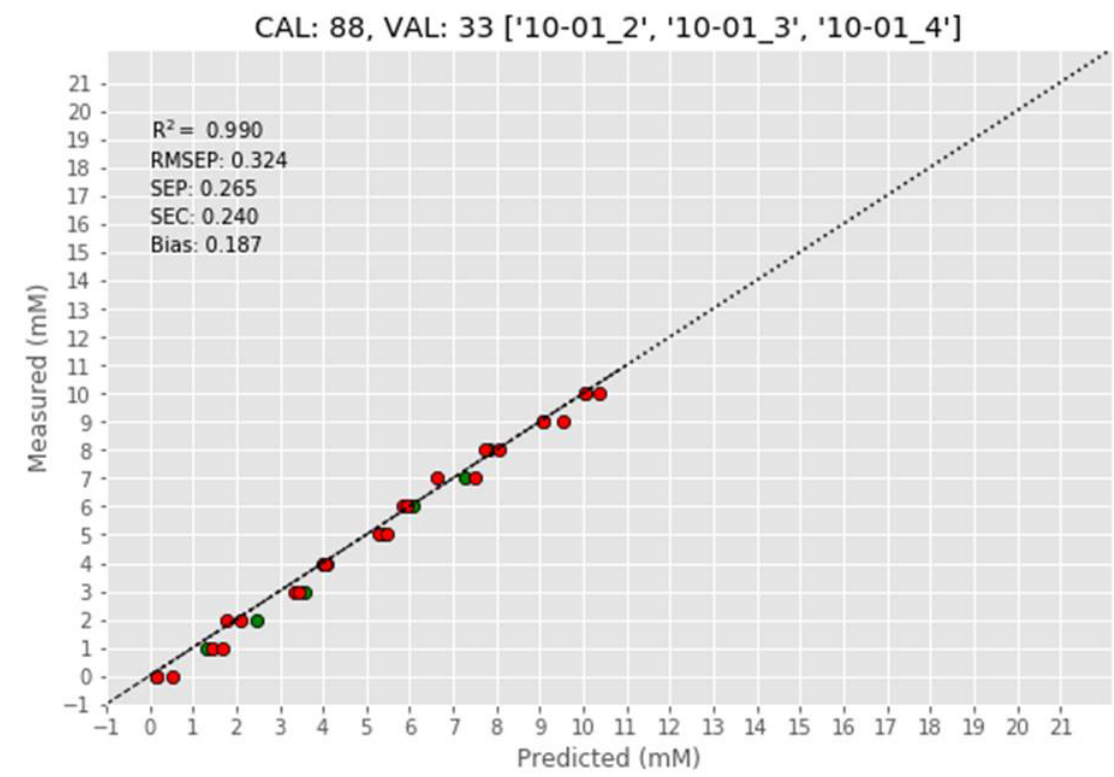

Figure 6. Sensor prediction performance for glucose embedded in a aqueous solution with $30 \mathrm{~g} / \mathrm{l}$ bovine serum albumin.

\section{OUTLOOK AND SUMMARY}

In this work we provided insight into the development of GaSb-based swept-wavelength laser technology and its application in the field of spectroscopic sensing of critical biomolecules. We presented extremely wide wavelength sweeps per single gain-chip of $600 \mathrm{~cm}^{-1}$ with a sweep rate of $180000 \mathrm{~cm}^{-1}$ which are promising for compact laser-based spectroscopic sensors used for real-time concentration level monitoring. Furthermore, we present initial sensor performance for glucose and a pathway to realize this sensor within silicon photonic integrated circuit technology and apply it for sensing in whole blood.

\section{REFERENCES}

[1] Pantelopoulos A., Bourbakis N. G., "A Survey on Wearable Sensor-Based Systems for Health Monitoring and Prognosis", IEEE Trans Syst Man Cybern B Applications and Reviews, Vol. 40, No, 1, pp. 1-12, (2010).

[2] K. Joon Kim Dong-Hee Shin, "An acceptance model for smart watches”, Internet Research, Vol. 25, No. 4, pp. 527$541,(2015)$.

[3] see https://www.wareable.com/fitness-trackers/best-heart-rate-monitor-and-watches

[4] Andru-Perez, J., Leff, D. R., Ip, H. M., Yang, G. Z., "From wearable Sensors to Smart Implants-Toward Pervasive and Personalized Healthcare", IEEE Trans Biomed Eng,m Vol. 62, No. 12, pp. 2750-2762, (2015).

[5] Vizbaras A., Šimonytė I., Miasojedovas A., Trinkūnas A., Bučiūnas T., Greibus M., Naujokaitė G., Torcheboeuf N., Droz S., Boiko D., Dambrauskas Ž, Gulbinas A, and Vizbaras K., "Swept-wavelength lasers based on GaSb gain-chip technology for non-invasive biomedical sensing applications in the 1.7-2.5 $\mu \mathrm{m}$ wavelength range," Biomed. Opt. Express 9, 4834-4849 (2018).

[6] Amerov, A. K., Chen, J., Arnold, M. A., "Molar Absorptivities of Glucose and other Biological Molecules in Aqueous Solutions over the First Overtone and Combination Regions of the Near-Infrared Spectrum", Applied Spectroscopy, Vol. 58, No. 10, pp. 1195-1204, (2004).

[7] Chen C., Xie Q., Yang D., Xiao H., Fu Y., Tan Y., Yao S., "Recent advances in electrochemical glucose biosensors: a review", RSC Advances, Vol. 3, No. 14, 4473, (2013). 
[8] Wang R., Malik A., Šimonytė I., Vizbaras A., Vizbaras K., and Roelkens G., „Compact GaSb/silicon-on-insulator 2.0x $\mu \mathrm{m}$ widely tunable external cavity lasers “, Optics Express, Vol. 24 No. 25, 28977-28986 (2016).

[9] Wang R, Vasiliev A., Muneeb M., Malik A., Sprengel S., Boehm G., Amann M. -C., Šimonyte I., Vizbaras A., Vizbaras K., Baets R., and Roelkens G., "III-V-on-Silicon Photonics Integrated Circuits for Spectroscopic Sensing in the 2-4 $\mu \mathrm{m}$ Wavelength Range,” Sensors, Vol. 17, No. 8, 1788 (2017). 